\title{
Oligonol suppresses lipid accumulation and improves insulin resistance in a palmitate-induced in HepG2 hepatocytes as a cellular steatosis model
}

\author{
Jae-Yeo Park ${ }^{1,2}$, Younghwa Kim³ ${ }^{3}$ Jee Ae $\mathrm{Im}^{4}$ and Hyangkyu Lee ${ }^{1,2^{*}}$
}

\begin{abstract}
Background: Oligonol is a low molecular weight form of polyphenol polymers derived from lychee fruits. Several studies suggest that Oligonol has an anti-obesity effect. Since obesity is tightly associated with insulin resistance, we investigated a possible remission effect of Oligonol on lipid accumulation and insulin resistance in human hepatic HepG2 cells.

Methods: HepG2 cells were treated with palmitate for $24 \mathrm{~h}$ to induce cellular hepatic steatosis and insulin resistance. The cells were then treated with Oligonol at subtoxic concentrations and examined for lipid metabolism, cytokine production, and insulin signaling using quantitative RT-PCR and western blot analysis.

Results: Oligonol treatment reversed the palmitate-induced intracellular lipid accumulation, down regulated the expression of lipogenic genes, and up-regulated genes for fatty acid degradation. Oligonol restored insulin sensitivity, as was determined by the phosphorylation states of IRS-1. Oligonol also inhibited STAT3-SOCS3 signaling and increased AMPK phosphorylation in HepG2 cells.

Conclusion: Oligonol treatment improved palmitate-induced cellular steatosis and insulin resistance in HepG2 cells with concomitant reduction of inflammatory cytokines and decrease in STAT3-SOCS3 and AMPK-mTOR pathways. Oligonol may have beneficial effects in lipid metabolism and insulin resistance in the liver.
\end{abstract}

Keywords: Oligonol, Hepatic steatosis, de novo fatty acid synthesis, Inflammation, Insulin resistance

\section{Background}

The mammalian liver controls the metabolic homeostasis for carbohydrates, lipids, and proteins. Functional failure of the organ is detrimental to an organism. Non-alcoholic fatty liver disease (NAFLD) is a broadly defined term for fatty liver-related diseases $[1,2]$. An early form of NAFLD is hepatic steatosis, characterized by the deposition of triglycerides in the liver as lipid droplets. Hepatic steatosis makes patients prone to more serious forms of NAFLD, such as non-alcoholic steatohepatitis (NASH) and liver cancer. Currently, there are not many therapeutic

\footnotetext{
* Correspondence: hkyulee@yuhs.ac

'Department of Clinical Nursing Science, Yonsei University College of

Nursing, 120-752 Seoul, Korea

${ }^{2}$ Nursing Policy and Research Institute, Biobehavioral Research Center, Yonsei University, 120-752 Seoul, Korea

Full list of author information is available at the end of the article
}

options for these diseases as the pathologies are not well understood [1].

The "two-hit hypothesis" has been proposed to understand the pathogenesis of NAFLD [3]. The "first hit" involves the deposition of triglycerides (TGs) and free fatty acids in hepatocytes, leading to the development of simple steatosis and insulin resistance, and the "second hit" involves hepatic injury, inflammation, and fibrosis, which are closely associated with oxidative stress in the liver $[4,5]$. Lipid peroxidation, production of inflammatory cytokines, and mitochondrial dysfunction are the attributes of inflammatory stress [6]. To study hepatic steatosis in vitro, a cellular hepatic model was previously established by treating human HepG2 hepatocarcinoma cells with free fatty acids (FFAs) [7]. Upon treatment with palmitate (PA), a saturated fatty acid, these cells show excess accumulation of cytosolic lipids [7], activation of lipogenic genes [8], 
and development of insulin resistance $[9,10]$, which are the cellular aspects of hepatic steatosis.

Oligonol is a low molecular weight oligomer of longchain polyphenols, typically proanthocyanidin found in various fruits, including lychees [11, 12]. Polyphenols derived from fruits and vegetables are known to function as anti-inflammatory and chemopreventive agents on various human diseases [13-15]. Despite its relatively recent introduction, Oligonol has been shown to display potential therapeutic effects on cancer, neurological, or metabolic disorders [11, 16]. Particularly, a randomized clinical trial showed that Oligonol reduces abdominal circumference and visceral fat, results in weight loss, and improves insulin resistance in healthy obese individuals [17]. Therefore, we hypothesized that Oligonol induced anti-inflammatory mechanisms and prevented the development of hepatic steatosis and liver damage.

The present study evaluated the effects of Oligonol in reducing hepatic steatosis in PA-treated HepG2 cells. This study showed that Oligonol suppressed de novo fatty acid synthesis and inflammatory cytokine production, which are associated with the inhibition of STAT3-SOCS3 and AMPK-mTOR.

\section{Methods}

\section{Cell culture and Oligonol treatment}

Human HepG2 cell line (a gift from Dr. E.-J. Lee at Yonsei University College of Medicine, Korea) was maintained in Hyclone Dulbecco's Modified Eagle's Medium (DMEM; Thermo Scientific, Rockford, IL, USA) containing $10 \%$ fetal bovine serum, $100 \mathrm{U} / \mathrm{mL}$ penicillin, and $100 \mu \mathrm{g} / \mathrm{mL}$ streptomycin. Cells were cultured in $5 \% \mathrm{CO}_{2}$ at $37{ }^{\circ} \mathrm{C}$. Oligonol (KCF Korea Co., Seoul, Korea) was dissolved in double-distilled $\mathrm{H}_{2} \mathrm{O}\left(\mathrm{ddH}_{2} \mathrm{O}\right)$, diluted in DMEM, and used to treat HepG2 cells. The final quantity of solvent did not exceed $0.1 \%$ of culture media for all experiments.

\section{MTT assay}

HepG2 cells were grown in 96-well plate with different concentrations of Oligonol for 24 or $48 \mathrm{~h}$. Cell viability was determined by colorimetry using the 3-(-4, 5dimethylthiazol-2-yl)-2, 5-diphenyltetrazolium bromide (MTT) (USB corporation, Cleveland, OH, USA; SigmaAldrich, Oakville, Canada). Insoluble formazan crystals were dissolved in DMSO and the absorbance was measured at $490 \mathrm{~nm}$ in a microplate reader (Molecular Devices, Mountain View, CA, USA).

\section{Preparation and treatment of sodium palmitate in HepG2 cells}

After reaching $80 \%$ confluence, the cells were cultured with fetal bovine serum-free medium containing $5 \%$ bovine serum albumin (BSA) overnight in a 6-well plate. Sodium palmitate (Sigma-Aldrich) was conjugated to fatty acid free-
BSA (Sigma-Aldrich). Briefly, $69.6 \mathrm{mg}$ sodium palmitate was dissolved in $1 \mathrm{ml}$ sterile water by alternating heating and vortexing at $70{ }^{\circ} \mathrm{C}$ to obtain $250 \mathrm{mM}$ stock solution. Immediately after the dissolution of PA, the stock solution was added to serum-free DMEM containing $5 \%$ fatty acidfree BSA to obtain $250 \mu \mathrm{M}$ PA solution. This solution was used to treat the cells [18]. Cells were treated with $200 \mu \mathrm{L}$ of $250 \mu \mathrm{M}$ PA or with different concentrations of Oligonol $(1,5$, or $10 \mu \mathrm{g} / \mathrm{ml})$ for $24 \mathrm{~h}$. Cells cultured in a medium containing $5 \%$ BSA were used as a control.

\section{Oil Red $O$ staining}

HepG2 cells grown in 6-well plates were collected, washed with phosphate buffered saline, and fixed with $4 \%$ paraformaldehyde for $1 \mathrm{~h}$. Cells were rinsed with $\mathrm{dd}_{2} \mathrm{O}$, dipped in $60 \%$ isopropanol for a few seconds, stained with Oil Red O (Sigma Chemical, St. Louis, MO, USA) for $10 \mathrm{~min}$, and rinsed with $\mathrm{dd}_{2} \mathrm{O}$ few times to remove the excess stain. Cell nuclei were stained with hematoxylin for a few seconds and washed with $\mathrm{ddH}_{2} \mathrm{O}$. Pictures were taken using an Axiovert 40CFL microscope (Olympus, Tokyo, Japan). For quantification of Oil Red O-based steatosis, HepG2 cells were cultured in a 96-well microplate at 5000 cells/well with PA plus/minus Oligonol for $24 \mathrm{~h}$ and Oil Red $\mathrm{O}$ staining was performed as described above. After washing and drying completely, $100 \mu \mathrm{L}$ of extraction solution was added to each well and the mixtures were incubated for $10 \mathrm{~min}$, followed by gentle vibration to release Oil Red O. The extraction solution was then transferred to another 96-well plate for measurement of optical density (OD) at $500 \mathrm{~nm}$ by microplate reader (Versamax; Molecular Devices Corporation, CA, USA). Tests were performed in triplicates.

\section{Quantitative real-time PCR (qRT-PCR)}

One microgram of total RNA purified with RNeasy Mini Kit (Qiagen, Valencia, CA, USA) was used to synthesize cDNA (Quanti Tech Reverse Transcription Kit; Qiagen). cDNA was amplified using SYBR Premix Ex Taq and genespecific primers (Table 1) in Takara Thermal Cycler Dice Real-Time system (Otsu, Shiga, Japan) $\left(95^{\circ} \mathrm{C}\right.$ for $5 \mathrm{~s}, 58^{\circ} \mathrm{C}$ for $10 \mathrm{~s}$, and $72{ }^{\circ} \mathrm{C}$ for $20 \mathrm{~s}$ for 40 cycles). All reactions were performed in triplicates and the data were normalized to glyceraldehyde 3-phosphate dehydrogenase (GAPDH).

\section{Western blot analysis}

Proteins were extracted from the cells using extraction buffer [ $50 \mathrm{mM}$ Tris $\mathrm{HCl}, \mathrm{pH}$ 8.0, $5 \mathrm{mM}$ EDTA, $150 \mathrm{mM}$ $\mathrm{NaCl}, 0.5 \%$ sodium deoxycholate, 1 \% Nonidet P-40, $0.1 \%$ SDS, $1 \mathrm{mM}$ PMSF, $1 \mathrm{mM} \mathrm{NaF}, 1 \mathrm{mM} \mathrm{NaVO}_{4}$, and protease inhibitor cocktail (Roche, Germany). Protein extracts were first separated on $10 \%$ polyacrylamide gels and electrophoretically transferred onto polyvinylidene fluoride membrane (Gelman Laboratory, Ann Arbor, MI, 
Table 1 Primer sequences used in this study

\begin{tabular}{|c|c|c|c|}
\hline Genes & Forward primer & Reveres primer & $\operatorname{Tm}\left(C^{\circ}\right)$ \\
\hline$\overline{A C C}$ & GAAGTCAGAGCCACGGCACA & GGCAATCTCAGTTCAAGCCAGTC & 62 \\
\hline FAS & AGCACTGCCTTCGGTTCAGTC & AAGAGCTGTGGAGGCCACTTG & 62 \\
\hline SREBP1C & CTCCGGCCACAAGGTACACA & GAGGCCCTAAGGGTTGACACAG & 60 \\
\hline HSL & CAAGTGTGTCAGCGCCTATGCT & GGGCTTTCTGGTCTGAGTTGGA & 64 \\
\hline PPARY & CTTGTGAAGGATGCAAGGGT & ATACAAATGCTITGCCAGGG & 62 \\
\hline CPT-1a & GGAATGAAATTCCCACTGTCTGTC & CAGTTCAGCCATCGCTGTTGTA & 60 \\
\hline $\mathrm{HADHa}$ & ACTTCCAAAGACACCAGTGCTTCA & GGCGCAAGACACCTGGTAGTATAGA & 60 \\
\hline LCAD & CCAGCTGCATGAAGCGAAAC & GCTGAACTCTGGCATCCACATAA & 60 \\
\hline UCP2 & TGTGCCCTTACCATGCTCCA & AGGGCTCGTTTCAGCTGCTC & 60 \\
\hline CYP2E1 & ATGTCTGCCCTCGGAGTGA & GATGTCCTTCCAGGTAGGTCC & 58 \\
\hline CYP4A11 & AGGAGCTCCAACAGGACCAG & CCTGATGGGTGAAGGCACAC & 68 \\
\hline PPARa & TCCTGAGCCATGCAGAATTTAC & AGTCTAAGGCCTCGCTGGTG & 60 \\
\hline TNFa & GACAAGCCTGTAGCCCATGTTGTA & CAGCCTTGGCCCTTGAAGA & 60 \\
\hline IL-6 & GTGGAGATTGTTGCCATCAACG & CAGTGGATGCAGGGATGATGTTCTG & 60 \\
\hline MCP-1 & AGCAGCAAGTGTCCCAAAGA & GGTGGTCCATGGAATCCTGA & 60 \\
\hline STAT3 & GGAATCCCGTGGGTTGCTTAC & TTGAATGCAGTGGCCAGGAC & 60 \\
\hline SOCS3 & CAGGAATGTAGCAGCGATGGAA & CCTGTCCAGCCCAATACCTGA & 60 \\
\hline GAPDH & TGAACGGGAAGCTCACTGG & TCCACCACCCTGTTGCTGTA & $58-64$ \\
\hline
\end{tabular}

USA). After blocking, the membranes were incubated with a primary antibody, and then with horseradish peroxidase-conjugated IgG (Santa Cruz Biotechnology, Santa Cruz, CA, USA). Blots were developed using ECL Detection Kit (Amersharm Pharmacia, UK). Primary antibodies to ACC, pACC, HSL, pHSL, FAS, STAT3, pSTAT3, IRS-1, pIRS-1(Ser363), AMPK, pAMPK, mTOR, pmTOR, S6K, pS6K were from Cell Signaling Technology (Denver, MA, USA), PPAR $\gamma$ and pIRS(Tyr632) were from Santa Cruz Biotechnology (Santa Cruz, CA, USA), SREBP-1 was from BD Biosciences (San Jose, CA, USA), and $\beta$-actin was from Sigma (St. Louis, MO, USA). Results of western blotting were normalized with those obtained for $\beta$ actin. Protein bands were quantified using densitometry with accompanying software (ImageJ).

\section{Free fatty acids and glycerol assay}

After treatment HepG2 cells with a various concentration of Oligonol in the presence of PA, free fatty acid and free glycerol contents in the cell supernatants was quantified using a free fatty acid or glycerol quantification kit according to the manufacturer's instructions (Biovision Inc., Milpitas, CA, USA). Released free fatty acid or glycerol was quantified at $570 \mathrm{~nm}$ on a 96-well plate reader (Molecular devices, Mountain View, CA, USA).

\section{Statistical analysis}

Data are presented as mean \pm SEM. Differences between the means of each group were analyzed using Student's $t$ test or one-way ANOVA test with Dunnett's multiple comparison test. $P$ values $<0.05$ were considered significant. The statistical software package Prism 5.0 (GraphPad Software, La Jolla, CA, USA) was used.

\section{Results}

Oligonol attenuated PA-induced intracellular lipid deposition in HepG2 cells

To assess the cytotoxic effects of Oligonol on HepG2 cells, the cells were treated with various concentrations of Oligonol $(0-50 \mu \mathrm{g} / \mathrm{ml})$ for 24 and $48 \mathrm{~h}$. Cell viability did not decrease below $90 \%$ of the control level after $48 \mathrm{~h}$, as determined by the MTT assay, indicating that the tested concentrations of Oligonol did not grossly affect cell growth and viability (Fig. 1a). Because the tested concentrations did not induce cytotoxicity, 1, 5, or $10 \mu \mathrm{g} / \mathrm{ml}$ of Oligonol was used for further studies.

Next, we investigated whether Oligonol inhibited PA-induced intracellular lipid accumulation in HepG2 cells [7]. The cells were incubated in $250 \mu \mathrm{M}$ PA and various concentrations of Oligonol for $24 \mathrm{~h}$, and intracellular lipid content was measured using oil red $\mathrm{O}$ staining. PA-treated HepG2 cells showed higher oil red O staining (Fig. $1 \mathrm{~b}$ and c) than vehicle-treated control cells, indicating higher TG content. Oligonol suppressed TG accumulation in HepG2 cells in a dose-dependent manner.

\section{Oligonol suppressed lipogenesis and enhanced lipolysis in PA-treated HepG2 cells}

Next, we examined the mechanisms underlying the inhibition of hepatic TG synthesis by Oligonol. Fatty 

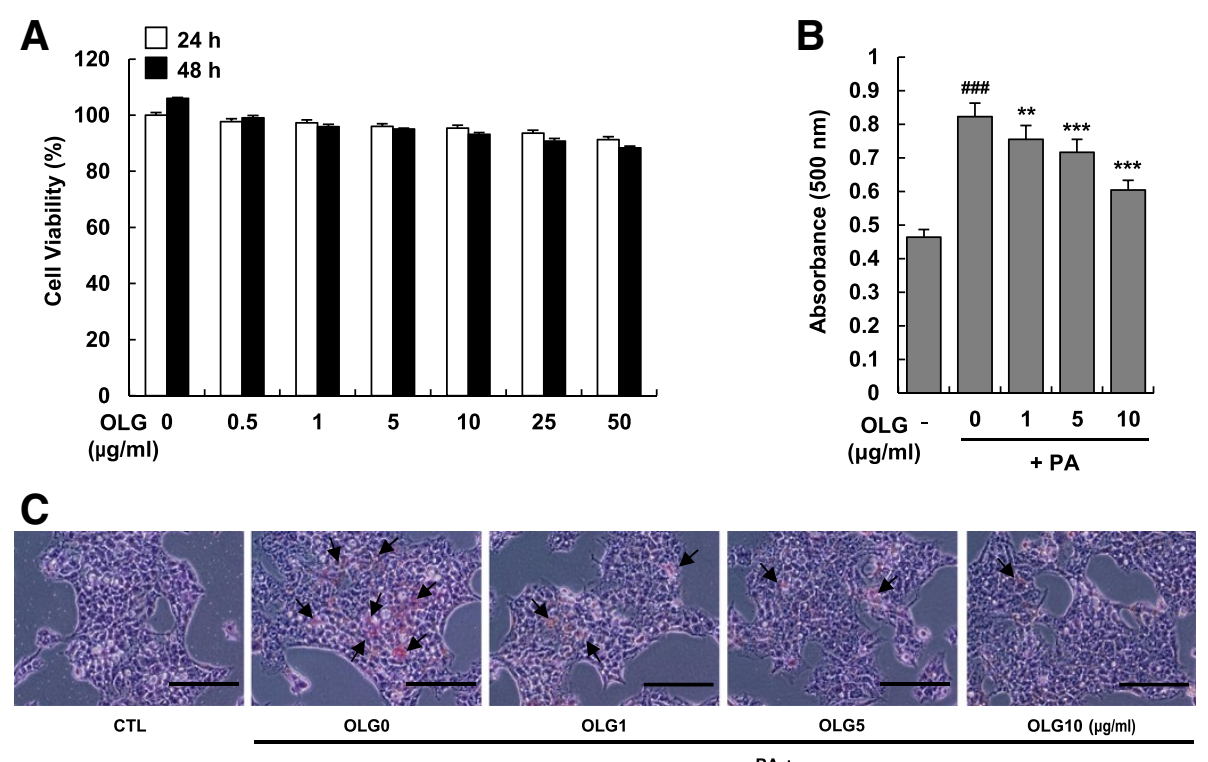

Fig. 1 Oligonol attenuated PA-induced increase in intracellular lipid content in HepG2 cells. (a) Oligonol cytotoxicity measurement. HepG2 cells were incubated with various concentrations of Oligonol (OLG; ranging from 0 to $50 \mu \mathrm{g} / \mathrm{ml}$ ) for 24 or $48 \mathrm{~h}$, and cell viability was measured by MTT assay. (b and c) Cells were treated with $250 \mu \mathrm{M}$ of palmitate (PA) in the absence or presence of Oligonol (ranging from 1 to $10 \mu \mathrm{g} / \mathrm{ml}$ ) for $24 \mathrm{~h}$, and stained with Oil-red O to visualize the intracellular lipid contents. (b) Lipid contents were quantified by its OD value at $500 \mathrm{~nm}$. Data were presented as mean \pm SEM of three independent experiments; each performed in triplicates. ${ }^{\# \#} P<0.005$ vs. vehicle-treated control; ${ }^{* *} P<0.01$, and ${ }^{* * *} P<0.005$ vs. palmitate-treated cells (PA). (c) Micrographs of phase contrast microscopy. Control (CTL) is vehicle treated cells. Scale bar is $100 \mu \mathrm{m}$

acid synthesis begins with acetyl-CoA and involves the addition of two carbon units to increase the length of the fatty acid chain. Acetyl-CoA carboxylase (ACC) converts acetyl-CoA to malonyl-CoA, which is a rate-limiting step in lipogenesis [19]. Fatty acid synthase (FAS) utilizes acetyl-CoA, malonyl-CoA, and NADPH, and elongates fatty acids chain to form PA [20]. Therefore, we examined the key enzymes and transcription factors involved in lipid metabolism in HepG2 cells. Oligonol suppressed the mRNA and protein expression of ACC, FAS, sterol regulatory element-binding protein-1 (SREBP-1), and peroxisome proliferator-activated receptor- $\gamma$ (PPAR $\gamma$ ) in a dose-dependent manner (Fig. 2a, b, and c), suggesting that Oligonol suppresses de novo fatty acid synthesis in HepG2 cells. Because Oligonol did not affect the mRNA levels of hormone sensitive lipase (HSL), we evaluated whether Oligonol regulated the activity of HSL by analyzing the levels of phosphorylated HSL in total cell lysates by performing western blotting. Phosphorylation of HSL at serine 660 was attenuated in PA-treated HepG2 cells, but all the 3 concentrations of Oligonol increased HSL phosphorylation. Further, we also found that phosphorylation of ACC at serine 79 was significantly increased in Oligonol-treated HepG2 cells (Fig. 2c).

Because Oligonol modulated the expression of genes involved in hepatic lipid metabolism, we examined whether the reduction in lipid accumulation was associated with lipolysis. HepG2 cells were incubated with Oligonol for
$24 \mathrm{~h}$, and the amounts of FFAs and glycerol in the culture medium were measured by performing a colorimetric assay. Oligonol increased the secretion of glycerol into the medium in a dose-dependent manner (Fig. 2d). In contrast, Oligonol did not increase the secretion of FFA into the medium and significantly decreased the intercellular levels of FFA (Fig. 2e).

\section{Oligonol increased fatty acid oxidation in PA-treated HepG2 cells}

Next, we examined whether Oligonol enhanced the degradation of fatty acids through $\beta$-oxidation. PA notably decreased the mRNA level of carnitine palmitoyltransferase 1a (CPT1a) in HepG2 cells, whereas Oligonol increased the mRNA level of CPT1a in a dose-dependent manner (Fig. 3a). PA had a similar effect on the level of long-chain L-3-hydroxyacyl-coenzyme A dehydrogenase $\alpha$ (HADH $\alpha)$. However, Oligonol had no effect on long-chain acyl-CoA dehydrogenase (LCAD) under the same condition. We also found that Oligonol attenuated PA-induced increase in the mRNA level of uncoupling protein 2 (UCP2), suggesting that Oligonol has antioxidant properties and protects hepatocytes from PA-induced lipotoxicity.

$\omega$-Oxidation, an alternative pathway of fatty acid metabolism, is activated upon failure of $\beta$-oxidation. Previous studies have shown that expression of the cytochrome P450 enzymes CYP2E1 and CYP4A11 is increased in patients with NAFLD [21] and that PA significantly 


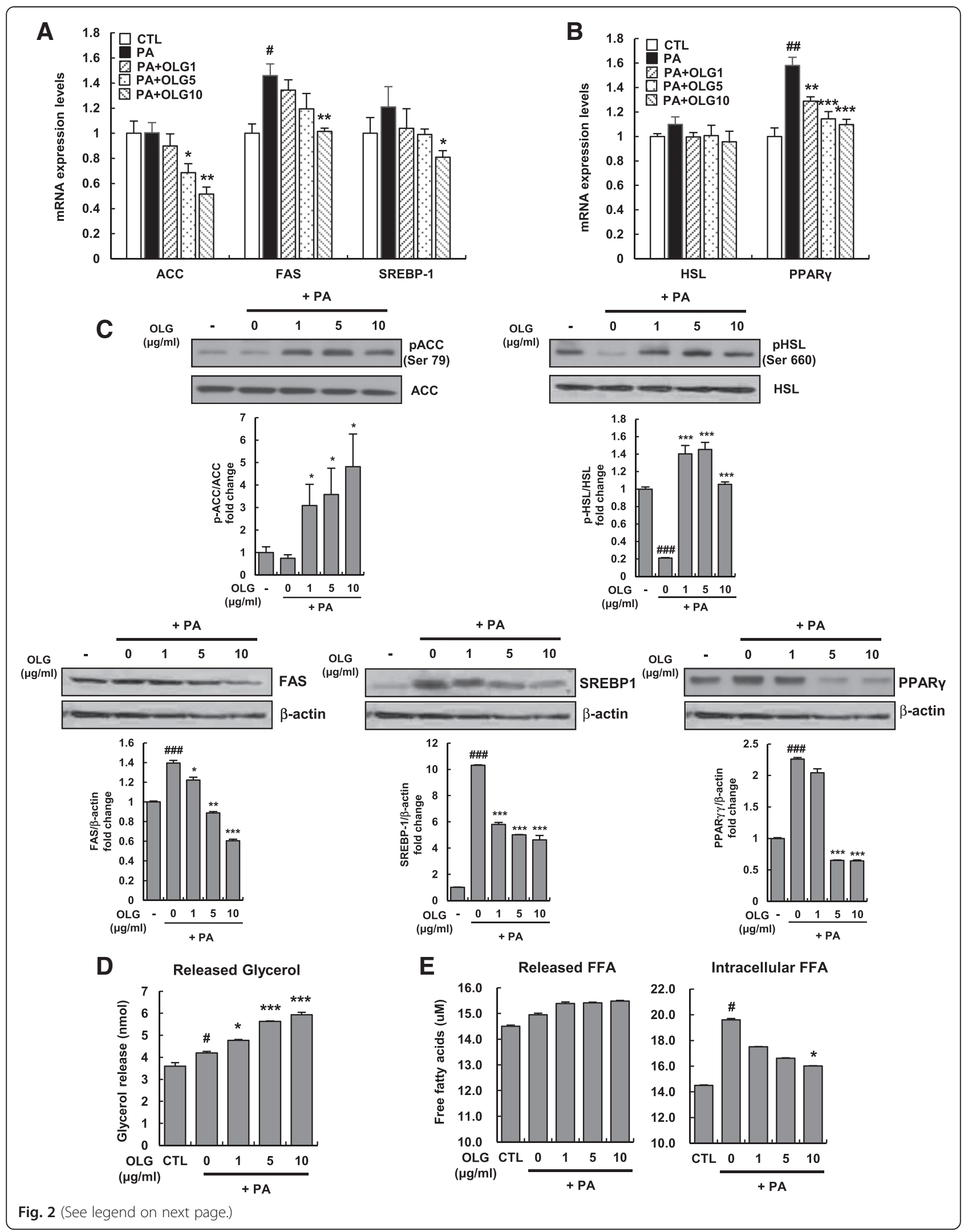


(See figure on previous page.)

Fig. 2 Oligonol suppressed lipogenesis while enhancing lipolysis in PA-treated HepG2 cells. HepG2 cells were treated with $250 \mu M$ of PA in the absence or presence of Oligonol (ranging from 1 to $10 \mu \mathrm{g} / \mathrm{ml}$ ) for $24 \mathrm{~h}$. (a) ACC, FAS, and SREBP-1c mRNA levels, (b) HSL and PPARY mRNA levels were measured by qRT-PCR. Values were normalized to GAPDH and presented as mean \pm SEM of three independent experiments; each performed in triplicates. White bars represent HepG2 cells treated with vehicle, and black bars represent HepG2 cells treated with PA. (c) Protein levels and phosphorylation states for these five genes were examined by Western blot analysis with appropriate antibodies. $\beta$-actin is a loading control. Graphs represent densitometric analysis of Western blots. (d and $\mathbf{e}$ ) Released glycerol and FFAs into the culture medium and intracellular FFA contents were quantified by its OD value at $570 \mathrm{~nm}$. Data were presented as mean \pm SEM of three independent experiments; each performed in triplicates. ${ }^{\prime} P<0.05$,

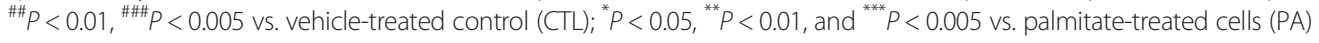

increases the level of CYP2E1 [22]. Therefore, we examined whether Oligonol inhibited the expression of these enzymes. Treatment of HepG2 cells with PA increased the mRNA levels of CYP2E1 and CYP4A11 by up to three fold; however, these levels were markedly reduced after Oligonol treatment.
Transcription factor PPAR $\alpha$ directly controls various steps of hepatic lipid metabolism, including expression of CPT1a and HADHa [23]. Because these genes are upregulated by Oligonol, we determined whether expression of PPAR $\alpha$ was also upregulated by Oligonol. Oligonol increased the mRNA level of PPAR $\alpha$ in a dose-
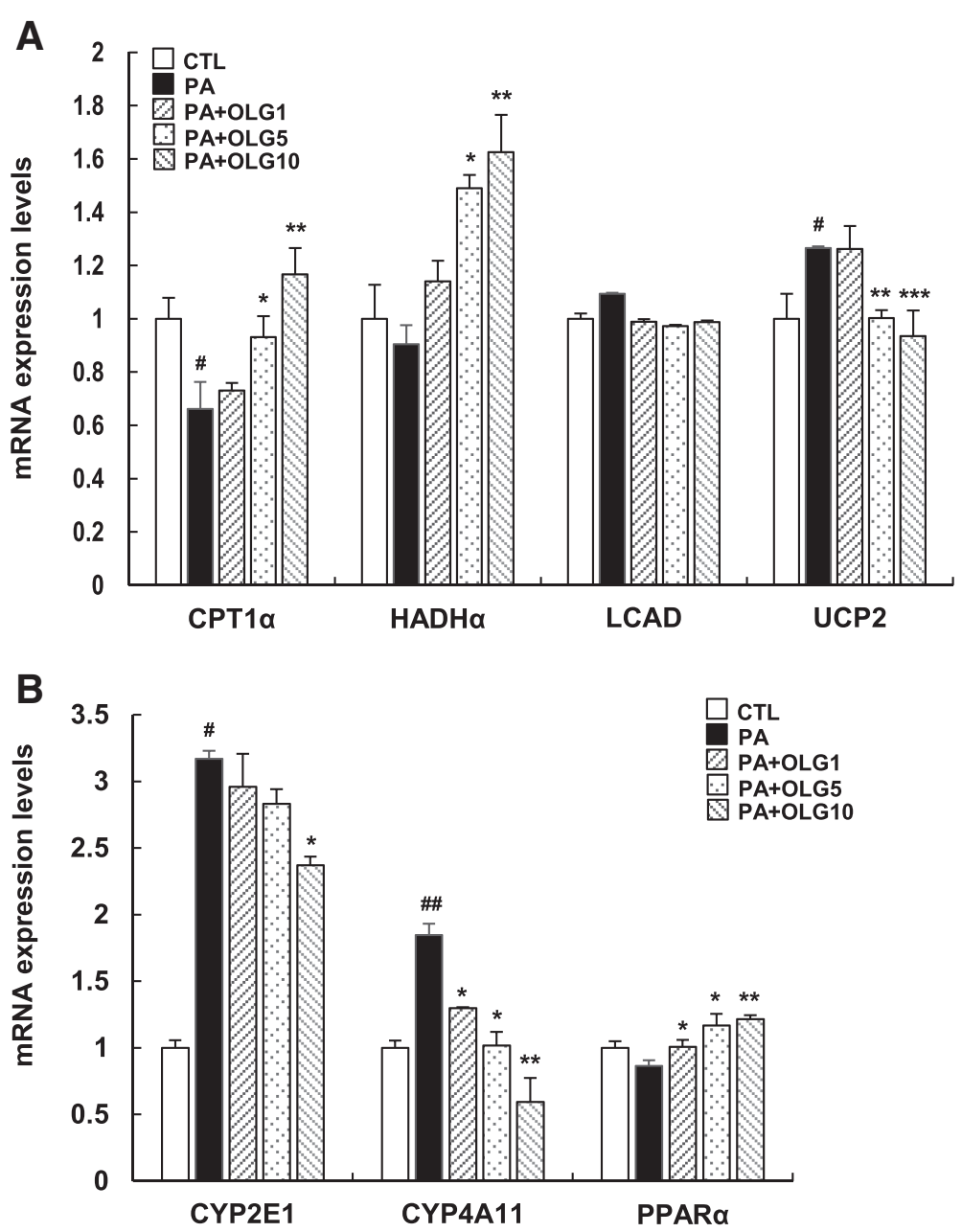

Fig. 3 Oligonol increased lipid oxidation in PA-treated HepG2 cells. HepG2 cells were treated with $250 \mu \mathrm{M}$ of PA in the absence or presence of Oligonol (ranging from 1 to $10 \mathrm{\mu g} / \mathrm{ml}$ ) for 24 h. (a) CPT1a, HADHa, LCAD, and UCP2 mRNA levels, (b) CYP2E1, CYP4A11, and PPARa mRNA levels were measured using qRT-PCR. Values were normalized to GAPDH. Data were presented as mean \pm SEM of three independent experiments; each performed in triplicates. White bars represent HepG2 cells treated with vehicle, and black bars represent HepG2 cells treated with PA. ${ }^{\# P}<0.05$, $\#^{\# \#} P<0.01$ vs. vehicle-treated control $(C T L) ;{ }^{*} P<0.05,{ }^{* *} P<0.01,{ }^{* * *} P<0.005$ vs. palmitate-treated cells (PA) 
dependent manner (Fig. 3b), indicating that Oligonol improves fatty acid catabolism at the transcriptional level at least in part by regulating the expression of PPAR $\alpha$.

\section{Oligonol suppressed PA-induced inflammation in HepG2 cells}

Chronic hepatic inflammation is tightly associated with the pathogenesis of NAFLD [24]. In fact, lipid peroxidation increases the production of inflammatory cytokines and mitochondrial dysfunction because $\beta$-oxidation generates increased levels of ROS, leading to oxidative stress $[5,25]$. To determine the effect of Oligonol on the mRNA expression of inflammatory cytokines, HepG2 cells were treated with Oligonol in the presence of PA. The mRNA expression of genes encoding inflammatory cytokines tumor necrosis factor $\alpha$ (TNF $\alpha$ ), interleukin 6 (IL-6), and monocytic chemotactic protein 1 (MCP-1) was significantly increased after PA treatment. However, Oligonol treatment at all the three concentrations decreased the expression of these inflammatory cytokines in a dose-dependent manner (Fig. 4a). The mRNA expression of IL-6 in PA-treated cells was $\sim 6$-fold higher than that in untreated cells. We measured the production and secretion of IL- 6 by performing enzyme-linked immunosorbent assay (ELISA) and found that Oligonol decreased the production IL-6, which was consistent with the decreased mRNA levels of IL-6 (Fig. 4b).

Because IL-6 directly activates signal transducer and activator of transcription 3 (STAT3), the effect of Oligonol on STAT3 signaling was tested by examining the mRNA levels of STAT3 and suppressor of cytokine signaling 3 (SOCS3). PA did not increase the mRNA expression of STAT3. Further, Oligonol suppressed the mRNA expression of STAT3 by up to $\sim 0.5$ fold. PA markedly increased the mRNA levels of SOCS3 by $\sim 2$ fold, which exerts a negative feedback control in response to the activation of STAT3 (Fig. 4c). Western blotting analysis of phosphoSTAT3 and STAT3 showed that PA increased the levels of phospho-STAT3 and that Oligonol decreased the levels of phospho-STAT3 at all the concentrations tested. Densitometric analysis showed no statistically significant decrease in the total level of STAT3. However, STAT3 level was decreased slightly, which was consistent with the decreased mRNA expression of STAT3 (Fig. 4d).

\section{Oligonol improved PA-induced insulin resistance}

Based on our finding that Oligonol suppressed IL-6STAT3-SOCS3 signaling in PA-treated HepG2 cells, we examined the effect of Oligonol on the key proteins involved in insulin signaling. We examined the changes in the phosphorylation of insulin receptor substrate-1 (IRS-1) in PA-treated HepG2 cells to assess insulin resistance. IRS-1 activity is either activated or inhibited by the phosphorylation of specific tyrosine or serine residues [26].
We first confirmed that PA treatment increased IRS-1 phosphorylation at serine 636 (an inhibitory site for insulin signaling) and decreased IRS-1 phosphorylation at tyrosine 632 (a stimulatory site for insulin signaling). Interestingly, Oligonol treatment reversed this phosphorylation pattern, i.e., phosphorylation at serine 636 was attenuated while that at tyrosine 632 was increased by all the 3 concentrations of Oligonol (Fig. 5a). To further confirm that insulin resistance was relieved in these cells, we examined the expression of genes involved in insulin resistance (INSR, CREB, CRTC, IGFBP-1, and GLUT2). No significant differences were observed in the expression of these genes between cells treated with Oligonol plus PA and untreated control cells (data not shown).

\section{Oligonol activated AMPK and inhibited mTOR-S6K pathway in HepG2 cells}

AMP-activated protein kinase (AMPK) is a critical enzyme for regulating hepatic lipogenesis and insulin resistance. Activated AMPK increases fatty acid oxidation, lipolysis, and insulin sensitivity in the skeletal muscle, liver, and adipose tissue $[18,27]$. To determine the modulation of AMPK by Oligonol, AMPK activation was examined by analyzing its phosphorylation levels in total cell lysates by Western blot analysis. All the concentrations of Oligonol equally enhanced the activity of AMPK, as determined by phosphorylation at threonine 172 compared with that in untreated control or PA-treated cells. Because AMPK negatively regulates mammalian target of rapamycin (mTOR) [28], we investigated whether Oligonol suppressed the activation of mTOR and its downstream component ribosomal protein S6 kinase (S6K). Western blotting analysis of mTOR and S6K showed that Oligonol markedly suppressed the phosphorylation of both mTOR and S6K in a dose-dependent manner. No differences were observed in the total protein levels of AMPK, mTOR, and S6K (Fig. 5b).

\section{Discussion}

Many polyphenols exert anti-obesity and hypolipidemic effects in animals and humans. These compounds have been investigated as complementary agents because of their potential beneficial effects on health and biosafety in various model systems [6, 29]. In the present study, we observed that Oligonol inhibited de novo fatty acid synthesis in HepG2 cells. We also found that Oligonol suppressed the expression of inflammatory cytokines such as IL-6, thus exhibiting anti-inflammatory effects, and regulated insulin resistance through the STAT3-SOCS3 and AMPK pathways. In the present study, we used PA, which is widely used to establish hepatic steatosis models that show increased FFA production [7]. Fatty acid metabolism in the liver involves the following steps: (1) de novo synthesis of fatty acids and uptake of plasma 


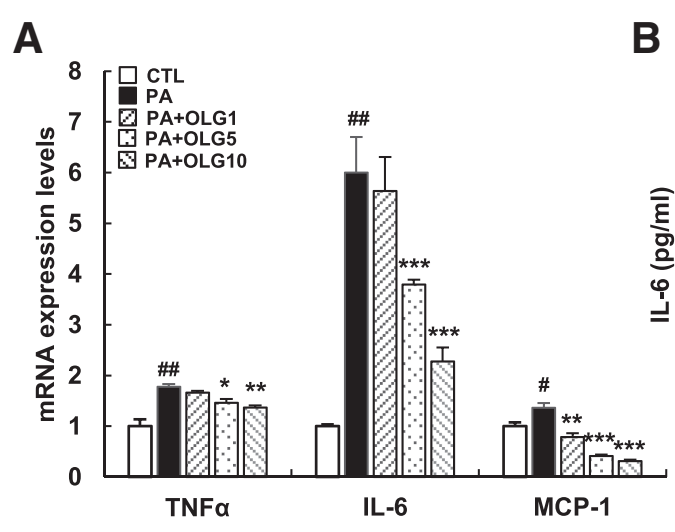

B
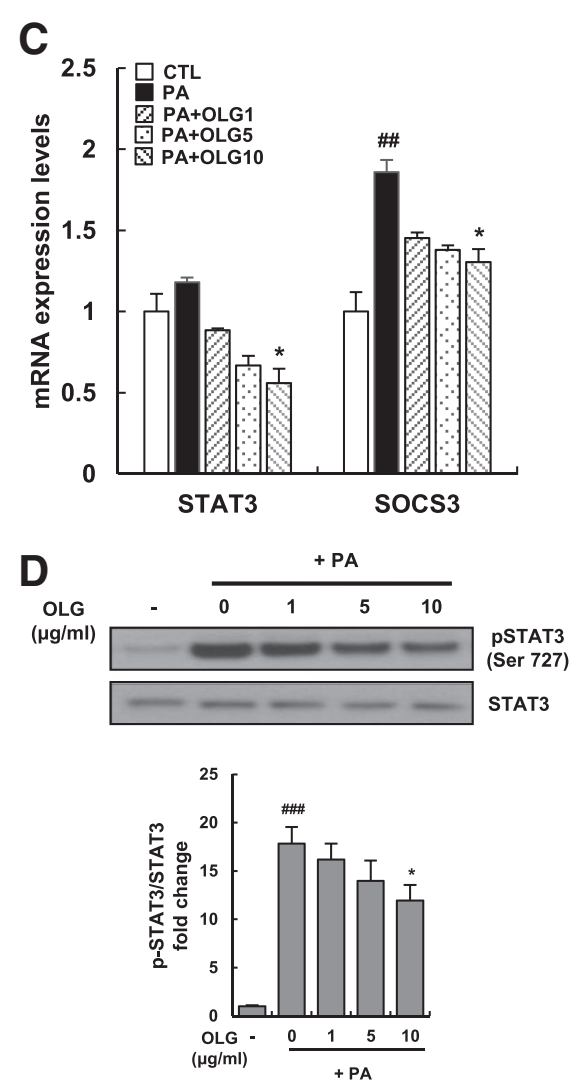

Fig. 4 Oligonol suppressed inflammation responses induced by PA in HepG2 cells. HepG2 cells were treated with $250 \mu M$ of PA in the absence or presence of Oligonol (ranging from 1 to $10 \mathrm{\mu g} / \mathrm{ml}$ ) for $24 \mathrm{~h}$. (a) TNFa, IL-6, and MCP-1 mRNA levels were measured by qRT-PCR. (b) IL-6 released into the culture medium was assayed. (c) STAT3 and SOCS3 mRNA levels were measured by qRT-PCR. Values were normalized to GAPDH. Data were presented as mean \pm SEM of three independent experiments; each performed in triplicates. White bars represent HepG2 cells treated with vehicle, and black bars represent HepG2 cells treated with PA. (d) STAT3 activation was examined by its phosphorylation state using Western blot analysis. $\beta$-actin was used as a loading control. Graphs represent densitometric analysis of Western blots. ${ }^{\#} P<0.05,{ }^{\# \#} P<0.01,{ }^{\# \# \#} P<0.005$ vs. vehicle-treated control $(C T L) ;{ }^{*} P<0.05,{ }^{* *} P<0.01$, and ${ }^{* * *} P<0.005$ vs. palmitate-treated cells (PA)

FFAs; (2) fatty acid oxidation in the mitochondria, peroxisomes, and microsomes; (3) neutralization of ROS generated through fatty acid oxidation; and (4) conversion of TGs to fatty acids [21]. Oligonol suppressed both ACC and FAS, which are the primary enzymes in lipogenesis. Oligonol also suppressed the mRNA expression of SREBP-1 and PPAR $\gamma$, indicating that this anti-lipogenic effect occurred at the transcriptional level. Lipogenesis is regulated by SREBP-1 and SREBP -2 . Particularly, SREBP-1 positively controls FFA metabolism by inducing the expression of target genes encoding ratelimiting enzymes such as ACC and FAS involved in de 


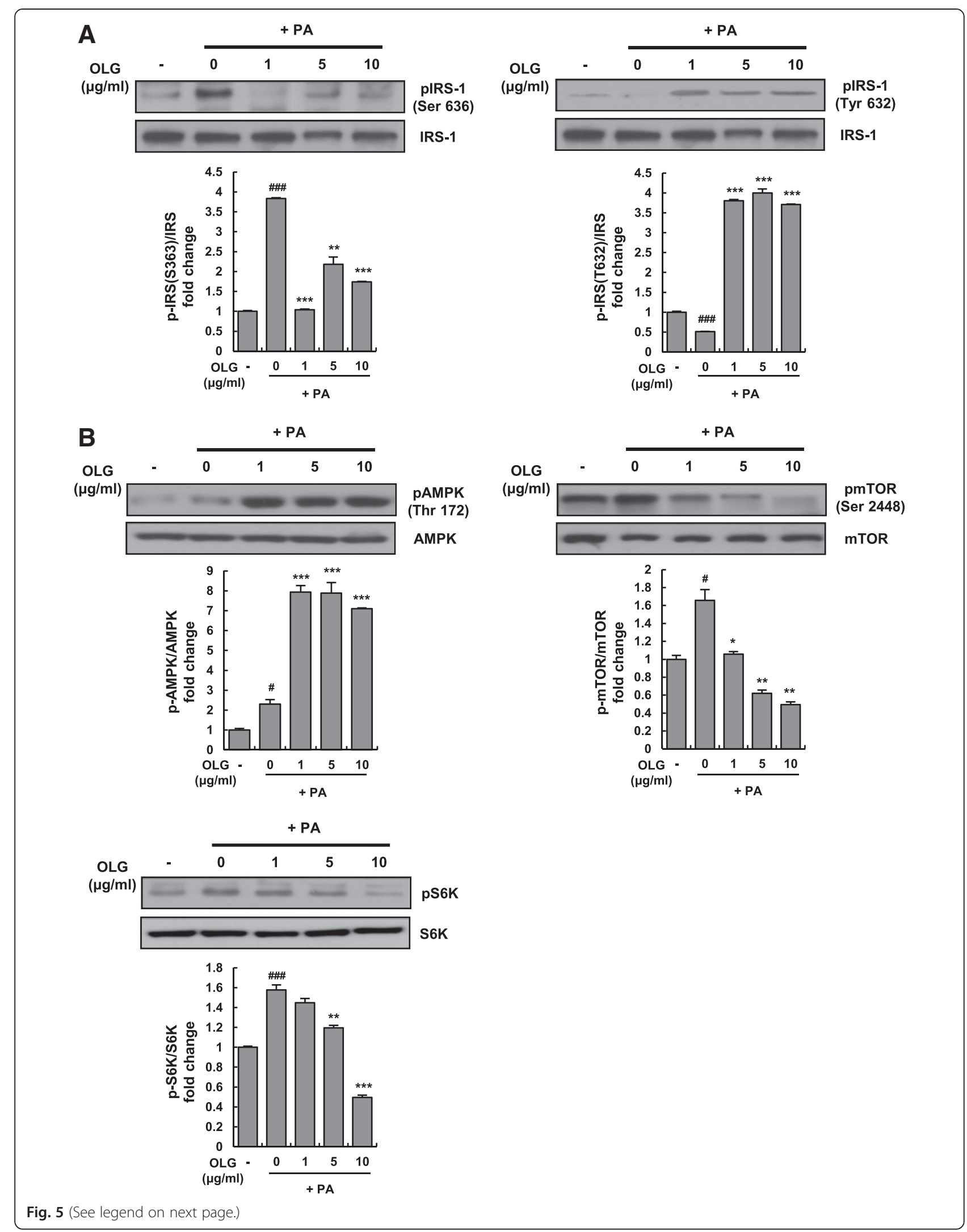


(See figure on previous page.)

Fig. 5 Oligonol attenuated PA-induced insulin resistance in HepG2 cells with concomitant activation of AMPK and suppression of the mTOR pathway. HepG2 cells were treated with $250 \mu \mathrm{M}$ of PA in the absence or presence of Oligonol (ranging from 1 to $10 \mu \mathrm{g} / \mathrm{ml}$ ) for $24 \mathrm{~h}$. The phosphorylation states of (a) IRS-1 or (b) AMPK, mTOR, and S6K were examined by Western blot analysis with appropriate antibodies. $\beta$-actin was used as a loading control. Graphs represent densitometric analysis of Western blots. ${ }^{\#} P<0.05$, ${ }^{\# \#} P<0.01$, \#\#\# $P<0.005$ vs. vehicle-treated control $(\mathrm{CTL}) ;{ }^{*} P<0.05,{ }^{* *} P<0.01$, and ${ }^{* * *} P<0.005$ vs. palmitate-treated cells (PA)

novo lipogenesis [30]. Oligonol activated the enzymes involved in lipid metabolism. Oligonol increased the phosphorylation of ACC and HSL (Fig. 2c). Phosphorylation of ACC at serine 79, a key phosphorylation site, inactivates ACC [31]. Thus, phosphorylation of ACC at serine 79 is a critical step in lipogenesis, indicating that this enzyme is a potential target for treating dyslipidemia [32]. Oligonol acts as a regulatory checkpoint in lipid synthesis by increasing phosphorylation. In fact, the mRNA expression of ACC, FAS, and SREBP-1 is increased in the livers of patients with NAFLD [21]. Moreover, liver-specific ACC1-knockout (LACC1KO) mice show decreased de novo concentrations of fatty acids and hepatic TGs [33].

Oligonol contributes to lipolysis by simultaneously inhibiting adipogenesis. Oligonol increases the release of glycerol from differentiated 3T3-L1adipocytes [34]. Moreover, Oligonol reduces fat content in the adipose tissues by increasing lipolysis both in vitro and in vivo $[35,36]$. Consistent with previous studies, Oligonol exerted a lipolytic effect on HepG2 cells in the present study, suggesting that Oligonol can efficiently regulate lipid metabolism in the adipose tissues and liver to prevent dyslipidemia. We further investigated the effect of Oligonol on the expression of genes associated with $\beta$-oxidation. Oxidation of fatty acids occurs mainly in the mitochondria. However, excessive fatty acid production in hepatocytes also activates alternative pathways such as $\beta$-oxidation in the peroxisomes and $\omega$-oxidation in the microsomes. In patients with NAFLD, mRNA expression of genes involved in fatty acid oxidation, such as $L C A D, H A D H \alpha, C Y P 2 E 1$, and CYP4A11, is increased while that of CPT1A and PPARA is decreased compared with that in the livers of normal individuals [21, 37]. PA, which is widely used to establish an in vitro model of fatty liver, mimicked dyslipidemic conditions by decreasing the mRNA levels of CPT1a and increasing the mRNA levels of CYP2E1 and CYP4A11. However, PA did not increase the mRNA levels of LCAD and HADH $\alpha$. Moreover, mRNA levels of HADH $\alpha$ increased after Oligonol treatment in a dose-dependent manner. Because Oligonol reduced the mRNA levels of CYP2E1 and CYP4A11, which were highly induced by PA, it is possible that acute PA stimulation may disrupt mitochondrial $\beta$-oxidation and alternatively induce $\omega$-oxidation. Thus, Oligonol reversed PA-induced expression of the above genes, suggesting that Oligonol facilitates the transport of long-chain fatty acids into the mitochondria and restores mitochondrial $\beta$-oxidation by increasing the mRNA expression of CPT1a and HADH $\alpha$.

Oligonol appears to eliminate excessive amounts of reactive oxygen species (ROS) produced during lipid oxidation in the mitochondria. UCP2 is a mitochondrial inner membrane carrier protein that mediates a proton leak across the inner membrane and uncouples mitochondrial electron transport from adenosine triphosphate (ATP) synthesis [38]. In many pathophysiological conditions such as NAFLD and obesity, UCP2 levels are elevated, indicating increased oxidative stress $[6,39]$. Our results showed that PA-induced UCP2 levels were inhibited by Oligonol, suggesting that Oligonol functions as an antioxidant and decreases the levels of ROS in HepG2 cells. However, this result was not consistent with the observation that Oligonol increased the gene expression of CPT1A and $H A D H \alpha$ and enhanced $\beta$-oxidation because increased $\beta$-oxidation induces ROS production. Therefore, it is conceivable that UCP2 levels may increase upon Oligonol treatment. However, UCP2 is not normally expressed in hepatocytes [40]; thus, the role of UCP2 in hepatocytes should be elucidated in further studies. Transcription of $U C P 2$ is regulated by other conditions such as energy expenditure and inflammation.

On the other hand, increase in the levels of inflammatory cytokines is associated with UCP2 expression. Incubation of hepatocytes with FFA increases the production of ROS and mRNA level of UCP2 [41], leading to oxidative stress. Overproduction of ROS also activates nuclear factor kappa beta (NF-kB) [42], a major transcription factor, that upregulates the expression of various cytokine such as TNF $\alpha$ and IL- 6 and contributes to mitochondrial dysfunction and increased ROS production. In fact, serum and hepatic levels of TNF $\alpha$, IL-1, and IL- 6 were elevated in a mouse model of NAFLD and patients with NAFLD [43-45]. Conversely, anti-cytokine antibodies decreased inflammation and prevented dyslipidemia and NAFLD in a mouse model $[46,47]$. Thus, it is possible that PA induces acute inflammation and expression of TNF $\alpha$ and IL- 6 in HepG2 cells. The above data indicate that these cytokines and not $\beta$-oxidation increase ROS production, leading to the upregulation of UCP2 mRNA levels. Thus, Oligonol may act as an antioxidant or anti-inflammatory agent to reduce the levels of $\mathrm{UCP} 2$.

Many studies have shown that hepatic steatosis is tightly associated with insulin resistance and obesity. A previous 
study reported that $40-100 \%$ obese people have comorbidities such as NAFLD and that $21-55 \%$ obese people have type 2 diabetes or hyperglycemia [48]. Increased levels of inflammatory cytokines play an important in the development of insulin resistance. TNF $\alpha$ was the first cytokine to be identified that impaired insulin action [49]. In obese rodent models and humans with NAFLD or obesity, a strong inverse correlation has been observed between serum TNF $\alpha$ levels and insulin-stimulated glucose metabolism [50, 51]. Although mechanisms involved in TNFo-induced insulin resistance are not completely understood, compelling evidence indicates that TNF $\alpha$ inhibits IRS-1- and IRS-2-mediated PI3-kinase activation, leading to insulin resistance [52]. IL-6 is another important cytokine involved in the regulation of insulin resistance. IL- 6 promotes insulin resistance by activating the STAT3-SOCS3 pathway in the liver [53]. Activation of STAT3 by phosphorylation subsequently increases SOCS3 expression through a negative feedback mechanism. SOCS3 then directly disrupts insulin signaling through ubiquitin-mediated degradation of IRS $[18,54]$. In the present study, Oligonol modulated reciprocal phosphorylation of IRS-1 by increasing the phosphorylation of the stimulatory residue tyrosine 632 and by decreasing the phosphorylation of the inhibitory residue serine 636, thus restoring insulin signaling. We further examined the expression levels of genes involved in insulin resistance. However, no changes were observed in the expression of these genes. To further understand the effects of Oligonol, a study involving a high-fat-induced obese mouse model should be performed to determine the comprehensive role of Oligonol in lipid metabolism.

In our previous study, Oligonol inhibited TG accumulation and lipogenesis by suppressing Akt-mTOR pathway in 3T3-L1 adipocytes [34]. Therefore, we tested whether Oligonol had the same anti-lipogenic effect on hepatic steatosis by using PA-treated HepG2 cells. Activated AMPK inhibits ATP-consuming processes such as synthesis of fatty acids or sterols and activates ATP-producing processes such as fatty acid oxidation [55]. Once activated through phosphorylation at threonine 172 residue in its $\alpha$ subunit, AMPK inhibits hepatic lipogenesis by phosphorylating ACC at the inhibitory serine 79 residue and by downregulating SREBP-1c [55]. Because Oligonol suppresses lipogenic gene expression and ACC activation (Fig. 2), we hypothesized that Oligonol activates AMPK and consequently inhibits lipogenic processes. As expected, Oligonol potently activated AMPK at all the tested concentrations and subsequently inhibited mTOR activation, thus inhibiting IRS-1 [26]. Interestingly, an anti-diabetic agent metformin, which activates AMPK in hepatocytes, exerted beneficial effects on lipid metabolism by suppressing the activity of ACC and reducing the expression of lipogenic enzymes [56]. Thus, the AMPK-mTOR pathway is a potential therapeutic target for treating hepatic steatosis. Oligonol showed a similar mechanism of action, indicating its beneficial effects in preventing insulin resistance and hepatic steatosis. IL-6 plays a paradoxical role in insulin signaling, which improves insulin sensitivity after physical exercise. Ruderman et al. reported that IL- 6 treatment of both muscle cells and adipocytes increased AMPK and ACC phosphorylation. Similar findings were observed in the muscle of mice after exercise. Moreover, these effects were decreased in IL-6 knockout mice [57]. IL-6 treatment improved insulin-induced glucose disposal and was accompanied by increased glucose transporter-4 (GLUT4) translocation to the plasma membrane and activation of AMPK [58]. However, IL-6-induced AMPK activation appears to be an acute response in skeletal muscle tissues after exercise. Low-grade chronic elevation of IL-6 is the primary cause of NAFLD. Thus, the prolonged effect of IL-6 on AMPK needs to be investigated further in hepatic steatosis models.

\section{Conclusions}

NAFLD is one of the most common causes of liver dysfunction. Excessive FFAs in the liver induce oxidative stress, inflammatory cytokine production, mitochondrial dysfunction, and insulin resistance, which all contribute to disease progression. In this study, we provided evidence that Oligonol has a protective effect on PA-induced cellular steatosis in HepG2 cells. Oligonol suppressed lipogenesis, while promoting fatty acid oxidation and lipolysis. PA-induced IL-6 production and STAT3 phosphorylation were significantly repressed by Oligonol. In addition, Oligonol inhibited mTOR phosphorylation by AMPK activation. Our results demonstrated that Oligonol may prevent hepatic steatosis by regulating AMPK-mTOR and STAT3-SOCS3 signaling pathways, and that these pathways are good therapeutic targets for treating hepatic dysfunction.

\section{Abbreviations \\ NAFLD: Non-alcoholic fatty liver disease; NASH: Non-alcoholic steatohepatitis; TG: Triglycerides; PA: Palmitate; FFAs: Free fatty acids; ACC: Acetyl-CoA carboxylase; FAS: Fatty acid synthase; SREBP-1: Sterol regulatory element- binding protein-1; PPARy: Peroxisome proliferator-activated receptor- $\gamma$; HSL: Hormone-sensitive lipase; CPT1a: Carnitine palmitoyltransferase 1a; LCAD: Long-chain acyl-CoA dehydrogenase; HADHa: Long-chain L-3- hydroxyacyl-coenzyme A dehydrogenase a; UCP2: Uncoupling protein 2; PPARa: Peroxisome proliferator-activated receptor-a; TNFa: Tumor necrosis factor a; IL-6: Interleukin 6; MCP: Monocytic chemotactic protein 1; STAT3: Signal transducer and activator of transcription 3; SOCS3: Suppressor of cytokine signaling 3, IRS-1, insulin receptor substrate-1; AMPK: AMP-activated protein kinase; mTOR: Mammalian target of rapamycin; S6K: Ribosomal protein S6 kinase; ROS: Reactive oxygen species; ATP: Adenosine triphosphate; NF-kB: Nuclear factor kappa beta; GLUT4: Glucose transporter-4.}

Competing interests

The authors declare that they have no competing interests.

\section{Authors' contribution}

JYP designed the experiment, conducted the experiments. JAI conceived the idea and supplied reagents. YK and HK designed the experiment, interpreted 
the experimental results, and wrote the manuscript. All authors contributed to manuscript preparations and approved the final manuscript.

\section{Acknowledgements}

This work was financially supported by the Faculty Research Assistance Program of Yonsei University College of Nursing (6-2013-0212), and the National Research Foundation of Korea Grant funded by the Korean Government (NRF-2012R1A12042620).

\section{Author details \\ ${ }^{1}$ Department of Clinical Nursing Science, Yonsei University College of Nursing, 120-752 Seoul, Korea. ${ }^{2}$ Nursing Policy and Research Institute, Biobehavioral Research Center, Yonsei University, 120-752 Seoul, Korea. ${ }^{3}$ Department of Emergency Medical Technology, Kyuongil University, 712-701 Gyeongsan, Kyungbook, Korea. ${ }^{4}$ Sport and Medicine Research Center, INTOTO Inc., 401 Dawoo BD, 90-6 Daeshin-Dong, 120-160 Seodaemun-Gu, Seoul, Korea.}

\section{Received: 11 July 2014 Accepted: 4 June 2015} Published online: 16 June 2015

\section{References}

1. Cohen J. Understanding HIV latency to undo it. Science. 2011;332(6031):786.

2. Michelotti GA, Machado MV, Diehl AM. NAFLD, NASH and liver cancer. Nat Rev Gastroenterol Hepatol. 2013;10(11):656-65.

3. Day CP, James OF. Steatohepatitis: a tale of two "hits"? Gastroenterology. 1998;114(4):842-5.

4. Sanyal AJ, Campbell-Sargent C, Mirshahi F, Rizzo WB, Contos MJ, Sterling RK, et al. Nonalcoholic steatohepatitis: association of insulin resistance and mitochondrial abnormalities. Gastroenterology. 2001;120(5):1183-92.

5. Browning JD, Horton JD. Molecular mediators of hepatic steatosis and liver injury. J Clin Invest. 2004;114(2):147-52.

6. Lin JK, Lin-Shiau SY. Mechanisms of hypolipidemic and anti-obesity effects of tea and tea polyphenols. Mol Nutr Food Res. 2006;50(2):211-7.

7. Gomez-Lechon MJ, Donato MT, Martinez-Romero A, Jimenez N, Castell JV, O'Connor JE. A human hepatocellular in vitro model to investigate steatosis. Chem Biol Interact. 2007;165(2):106-16.

8. Liu JF, Ma Y, Wang Y, Du ZY, Shen JK, Peng HL. Reduction of lipid accumulation in HepG2 cells by luteolin is associated with activation of AMPK and mitigation of oxidative stress. Phytother Res. 2011;25(4):588-96.

9. Gao D, Nong S, Huang X, Lu Y, Zhao H, Lin Y, et al. The effects of palmitate on hepatic insulin resistance are mediated by NADPH Oxidase 3-derived reactive oxygen species through JNK and p38MAPK pathways. J Biol Chem 2010;285(39):29965-73.

10. Jung TW, Choi HY, Lee SY, Hong HC, Yang SJ, Yoo HJ, et al. Salsalate and Adiponectin Improve Palmitate-Induced Insulin Resistance via Inhibition of Selenoprotein P through the AMPK-FOXO1alpha Pathway. PLOS ONE. 2013;8(6), e66529.

11. Aruoma OI, Sun B, Fujii H, Neergheen VS, Bahorun T, Kang KS, et al. Low molecular proanthocyanidin dietary biofactor Oligonol: Its modulation of oxidative stress, bioefficacy, neuroprotection, food application and chemoprevention potentials. Biofactors. 2006;27(1-4):245-65.

12. Fujii $H$, Sun B, Nishioka $H$, Hirose A, Aruoma Ol. Evaluation of the safety and toxicity of the oligomerized polyphenol Oligonol. Food Chem Toxicol. 2007;45(3):378-87.

13. Stoclet JC, Chataigneau T, Ndiaye M, Oak MH, El Bedoui J, Chataigneau M et al. Vascular protection by dietary polyphenols. Eur J Pharmacol. 2004;500(1-3):299-313.

14. Lau FC, Shukitt-Hale B, Joseph JA. The beneficial effects of fruit polyphenols on brain aging. Neurobiol Aging. 2005;26 Suppl 1:128-32

15. Gonzalez-Gallego J, Garcia-Mediavilla MV, Sanchez-Campos S, Tunon MJ. Fruit polyphenols, immunity and inflammation. Br J Nutr. 2010;104 Suppl 3:S15-27.

16. Ogasawara J, Kitadate K, Nishioka H, Fujii H, Sakurai T, Kizaki T, et al. Comparison of the effect of oligonol, a new lychee fruit-derived low molecular form of polyphenol, and epigallocatechin-3-gallate on lipolysis in rat primary adipocytes. Phytother Res. 2011:25(3):467-71.

17. Nishihira J, Sato-Ueshima M, Kitadate K, Wakame K, Fujii H. Amelioration of abdominal obesity by low-molecular-weight polyphenol (Oligonol) from lychee. J Funct Foods. 2009;1(4):341-8.
18. Mayer CM, Belsham DD. Palmitate attenuates insulin signaling and induces endoplasmic reticulum stress and apoptosis in hypothalamic neurons: rescue of resistance and apoptosis through adenosine $5^{\prime}$ monophosphate-activated protein kinase activation. Endocrinology. 2010;151(2):576-85.

19. Munday MR. Regulation of mammalian acetyl-CoA carboxylase. Biochem Soc Trans. 2002;30(Pt 6):1059-64.

20. Jensen-Urstad AP, Semenkovich CF. Fatty acid synthase and liver triglyceride metabolism: housekeeper or messenger? Biochim Biophys Acta. 2012;1821(5):747-53.

21. Kohjima M, Enjoji M, Higuchi N, Kato M, Kotoh K, Yoshimoto T, et al. Re-evaluation of fatty acid metabolism-related gene expression in nonalcoholic fatty liver disease. Int JMol Med. 2007;20(3):351-8.

22. Raucy JL, Lasker J, Ozaki K, Zoleta V. Regulation of CYP2E1 by ethanol and palmitic acid and CYP4A11 by clofibrate in primary cultures of human hepatocytes. Toxicol Sci. 2004;79(2):233-41.

23. Rakhshandehroo M, Knoch B, Muller M, Kersten S. Peroxisome proliferator-activated receptor alpha target genes. PPAR Res 2010, 2010

24. Asrih M, Jornayvaz FR. Inflammation as a potential link between nonalcoholic fatty liver disease and insulin resistance. J Endocrinol. 2013;218(3):R25-36.

25. Fromenty B, Robin MA, Igoudjil A, Mansouri A, Pessayre D. The ins and outs of mitochondrial dysfunction in NASH. Diabetes Metab. 2004;30(2):121-38.

26. Gual P, Le Marchand-Brustel Y, Tanti JF. Positive and negative regulation of insulin signaling through IRS-1 phosphorylation. Biochimie. 2005:87(1):99-109.

27. Thompson MP, Kim D. Links between fatty acids and expression of UCP2 and UCP3 mRNAs. FEBS Lett. 2004;568(1-3):4-9.

28. Marshall S. Role of insulin, adipocyte hormones, and nutrient-sensing pathways in regulating fuel metabolism and energy homeostasis: a nutritional perspective of diabetes, obesity, and cancer 2006. Sci STKE. 2006;346:re7.

29. Meydani M, Hasan ST. Dietary polyphenols and obesity. Nutrients. 2010;2(7):737-51.

30. Horton JD, Goldstein JL, Brown MS. SREBPs: activators of the complete program of cholesterol and fatty acid synthesis in the liver. J Clin Invest. 2002;109(9):1125-31.

31. Ha J, Daniel S, Broyles SS, Kim KH. Critical phosphorylation sites for acetyl-CoA carboxylase activity. J Biol Chem. 1994;269(35):22162-8.

32. Postic C, Girard J. Contribution of de novo fatty acid synthesis to hepatic steatosis and insulin resistance: lessons from genetically engineered mice. J Clin Invest. 2008;118(3):829-38.

33. Mao J, DeMayo FJ, Li H, Abu-Elheiga L, Gu Z, Shaikenov TE, et al. Liver-specific deletion of acetyl-CoA carboxylase 1 reduces hepatic triglyceride accumulation without affecting glucose homeostasis. Proc Natl Acad Sci U S A. 2006;103(22):8552-7.

34. Park JY, Kim Y, Im JA, You S, Lee H. Inhibition of Adipogenesis by Oligonol through Akt-mTOR Inhibition in 3 T3-L1 Adipocytes. Evid Based Complement Alternat Med. 2014:2014:895272

35. Ogasawara J, Kitadate K, Nishioka H, Fujii H, Sakurai T, Kizaki T, et al. Oligonol, a new lychee fruit-derived low-molecular form of polyphenol, enhances lipolysis in primary rat adipocytes through activation of the ERK1/2 pathway. Phytother Res. 2009;23(11):1626-33.

36. Sakurai T, Nishioka H, Fujii H, Nakano N, Kizaki T, Radak Z, et al. Antioxidative effects of a new lychee fruit-derived polyphenol mixture, oligonol, converted into a low-molecular form in adipocytes. Biosci Biotechnol Biochem. 2008;72(2):463-76.

37. Nakamuta M, Kohjima M, Morizono S, Kotoh K, Yoshimoto T, Miyagi I, et al. Evaluation of fatty acid metabolism-related gene expression in nonalcoholic fatty liver disease. Int J Mol Med. 2005;16(4):631-5.

38. Krauss S, Zhang CY, Lowell BB. A significant portion of mitochondrial proton leak in intact thymocytes depends on expression of UCP2. Proc Natl Acad Sci U S A. 2002;99(1):118-22.

39. Millet $L$, Vidal $H$, Andreelli $F$, Larrouy D, Riou JP, Ricquier D, et al. Increased uncoupling protein-2 and -3 mRNA expression during fasting in obese and lean humans. J Clin Invest. 1997;100(11):2665-70.

40. Shang Y, Liu Y, Du L, Wang Y, Cheng X, Xiao W, et al. Targeted expression of uncoupling protein 2 to mouse liver increases the susceptibility to lipopolysaccharide/galactosamine-induced acute liver injury. Hepatology. 2009:50(4):1204-16.

41. Cortez-Pinto H, Zhi Lin H, Qi Yang S, Odwin Da Costa S, Diehl AM. Lipids up-regulate uncoupling protein 2 expression in rat hepatocytes. Gastroenterology. 1999;116(5):1184-93. 
42. Garcia-Ruiz C, Colell A, Morales A, Kaplowitz N, Fernandez-Checa JC. Role of oxidative stress generated from the mitochondrial electron transport chain and mitochondrial glutathione status in loss of mitochondrial function and activation of transcription factor nuclear factor-kappa B: studies with isolated mitochondria and rat hepatocytes. Mol Pharmacol. 1995;48(5):825-34.

43. Dowman JK, Tomlinson JW, Newsome PN. Pathogenesis of non-alcoholic fatty liver disease. QJM. 2010;103(2):71-83.

44. Tarantino G, Savastano S, Colao A. Hepatic steatosis, low-grade chronic inflammation and hormone/growth factor/adipokine imbalance. World J Gastroenterol. 2010;16(38):4773-83.

45. Haukeland JW, Damas JK, Konopski Z, Loberg EM, Haaland T, Goverud I, et al. Systemic inflammation in nonalcoholic fatty liver disease is characterized by elevated levels of CCL2. J Hepatol. 2006:44(6):1167-74.

46. Li Z, Yang S, Lin H, Huang J, Watkins PA, Moser AB, et al. Probiotics and antibodies to TNF inhibit inflammatory activity and improve nonalcoholic fatty liver disease. Hepatology. 2003;37(2):343-50.

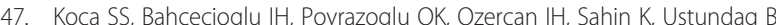
The treatment with antibody of TNF-alpha reduces the inflammation, necrosis and fibrosis in the non-alcoholic steatohepatitis induced by methionine- and choline-deficient diet. Inflammation. 2008;31(2):91-8.

48. James OF, Day CP. Non-alcoholic steatohepatitis (NASH): a disease of emerging identity and importance. J Hepatol. 1998;29(3):495-501.

49. Lang CH, Dobrescu C, Bagby GJ. Tumor necrosis factor impairs insulin action on peripheral glucose disposal and hepatic glucose output. Endocrinology. 1992;130(1):43-52

50. Tilg $\mathrm{H}$. The role of cytokines in non-alcoholic fatty liver disease. Dig Dis. 2010;28(1):179-85.

51. Arner P. The adipocyte in insulin resistance: key molecules and the impact of the thiazolidinediones. Trends Endocrinol Metab. 2003;14(3):137-45.

52. del Aguila LF, Claffey KP, Kirwan JP. TNF-alpha impairs insulin signaling and insulin stimulation of glucose uptake in C2C12 muscle cells. Am J Physiol. 1999;276(5 Pt 1):E849-55.

53. Qatanani M, Lazar MA. Mechanisms of obesity-associated insulin resistance: many choices on the menu. Genes Dev. 2007;21(12):1443-55

54. Rui HL, Fan E, Zhou HM, Xu Z, Zhang Y, Lin SC. SUMO-1 modification of the C-terminal KVEKVD of Axin is required for JNK activation but has no effect on Wnt signaling. J Biol Chem. 2002:277(45):42981-6.

55. Zhou G, Sebhat IK, Zhang BB. AMPK activators-potential therapeutics for metabolic and other diseases. Acta Physiol. 2009;196(1):175-90.

56. Zhou G, Myers R, Li Y, Chen Y, Shen X, Fenyk-Melody J, et al. Role of AMP-activated protein kinase in mechanism of metformin action. J Clin Invest. 2001;108(8):1167-74

57. Ruderman NB, Keller C, Richard AM, Saha AK, Luo Z, Xiang X, et al. Interleukin-6 regulation of AMP-activated protein kinase. Potential role in the systemic response to exercise and prevention of the metabolic syndrome. Diabetes. 2006:55(2):S48-54.

58. Carey AL, Steinberg GR, Macaulay SL, Thomas WG, Holmes AG, Ramm G, et al. Interleukin-6 increases insulin-stimulated glucose disposal in humans and glucose uptake and fatty acid oxidation in vitro via AMP-activated protein kinase. Diabetes. 2006:55(10):2688-97.

\section{Submit your next manuscript to BioMed Central and take full advantage of:}

- Convenient online submission

- Thorough peer review

- No space constraints or color figure charges

- Immediate publication on acceptance

- Inclusion in PubMed, CAS, Scopus and Google Scholar

- Research which is freely available for redistribution

Submit your manuscript at www.biomedcentral.com/submit 